PULSKA AKADEMIA NAUK-ZAKŁAD BADANIA SSAKOW
A C $\mathrm{T}$ A
T H E R
O L O G I C A
VOI. III, 5.
BIAŁOWIEŻA
15. XI. 1959

Jerzy SI D O R O W I Z

\title{
Über Morphologie und Biologie der Haselmaus (Muscardinus avellanarius L.) in Polen
}

\author{
Moriologia i biologia orzesznicy \\ (Muscardinus avellanarius L.) w Polsce
}

\section{EINFUHRUNG}

Nach Angaben von Skuratowicz (1947) und Van den Brink (1956) tritt der kleinste Vertreter der einheimischen Muscardinae - die Haselmaus grundsätzlich in ganz Polen auf, aber ihre genaue Verbreitung ist nicht genau bekannt. Die Haselmaus wurde in folgenden Gebieten Polens gefangen: Nordostkarpaten, Rytro, Jordanów, Pieniny, Bieszczady - Umgegend von Zamość, Urwald von Sandomierz und Łysa-Góra (Polnische Platte). Zahlreicher tritt sie aber in der Kampinos-Heide (Dziekanow), Umgegend von Warszawa auf. Karpiński (1954) gibt das Auftreten dieser Art im Urwald von Białowieża an. Für den Teil des auf dem Gebiet der U.d.S.S.R. liegenden Urwaldes gibt Angaben Turov (1955). Beide letztgenennten Autoren geben jedoch keine Fangorte dieses Tieres an. Trotz 12-jähriger Fangtätigkeit im Gelände des Białowieża Nationalparkes stelite man hier keine Anwesenheit der Haselmaus fest, obwohl man ab und zu Baumschläfer und Siebenschläfer gefangen hatte.

Es fehlt an Mitteilungen über Anwesenheit der Haselmaus auf dem Gebiet von West- und Nordwest-Polen. Die Festlegung der nördlichen Grenze dieses Nagers wie auch des Baumschläfers und des Siebenschläfers scheint ein interessantes Problem darzustellen, welches eine gewisse Aufklärung auf die Regrenzungsfaktoren des Auftretens nach Norden hin geben kann, umsomehr als es sich hier vielmehr um südliche Arten handelt. Nebenbei sei bemerkt, lass die Haselmaus den am weitesten nach Norden anzutreffenden Vertre- 
ter der Muscardinae darstellt. (In England tritt auch der Siebenschläfer auf, welcher dort ein eingeführtes Tier ist).

In der polnischen theriologischen Literatur finden wir die ersten Mitteilungen über die Haselmaus bei Tyzenhauz: "In Litauen seltene". Stronczyński (1839) gibt einige Einzelheiten aus der Biologie dieses Tieres an. B elke (1848) schreibt: „Sie lebt in Haselnusshainen, sie wird zwar selten aber auch in Kiefernwäldern angetroffen ein in Polen und Litauen ziemlich rares Tier".

Pietruski $(1853,1869)$ nennt sie Myoxus avellanarius; er gibt eine ganze Reihe von Einzelheiten aus dem Leben der Haselmaus, welche bis heute an ihrer Aktualität nichts verloren haben. Dieser Autor hielt Haselmäuse, aber diese vermehrten sich in der Gefąngenschaft nicht.

Eine Reihe von Autoren gab später faunistische Notizen über das Vorkommen der Haselmaus, vor allem im Gebirge und Vorgebirge. Die Mehrheit verbindet ihr Auftreten mit dem Vorhandensein von Haselnussträuchern (Corylus avellana) im betreffenden Gebiet und unterstreicht die Seltenheit dieser Art.

Skuratowicz (1948) veröffentlicht eine Anzahl von Nachrichten zur I.ebensweise dieses Nagers, indem er Winter- und Sommernester und Biotope beschreibt.

Die Angaben aus dem Schrifttum zusammenfassend, kann man feststellen, dass wenig Material üher die Morphologie der Haselmaus vorhanden ist. Unbekannt ist die westliche und nördliche Grenze dieser Art in Polen, ausserdem fehlt es an systematisch gesammelten Beobachtungen aus der Biologie und Ökologie (Vermehrung, Winterschlaf, Haarwechsel, Nahrung, Zyklus der Tagesaktivität, Zyklus der jahreszeitlichen Aktivität u.s.w.).

Die Haselmaus kann ein interessantes Obiekt für Untersuchungen über den Winterschlaf darstellen. Im Schrifttum findet man aber nur sehr wenige Arbeiten zu diesem Thema ( $\mathrm{E}$ is entra ut, 1929; Tomilin, 1958).

Der Zweck meiner Arbeit liegt im Versuch einige Liicken, aber besonders auf dem Gebiet der Morphologie auszufüllen, wenn es sich um unsere Kenntnis dieser Art in Polen handelt.

An dieser Stelle möchte ich den Kollegen aus dem Laboratorium für Ökologie der Polnischen Akademie der Wissenschaften, meinen Dank sagen aber besonders dem Leiter der Geländestation für Ökologie in Dziekanów Herrn mgr R. Andrzejewski für die Bereitstellung seines Materials und Mithilfe bei der Einsammlung des Materiales in der Kampinos-Heide.

\section{MATERIAL UND METHODE}

Die vorliegende Arbeit stützt sich auf das in der Kampinos-Heide eingefangene Material (Bälge befinden sich in den Sammlungen des Institutes für Säugetierforschung der P. A. d. W. in Białowieża) und auf eine Serie von Exemplaren aus Mokuszyn (Rokitnosümpfe, Belorussische S. S. R.), welche durch A. D e h nel gesammelt, und zu dieser Arbeit aus dem Zoologischen Institut der P. A. d. W. in War- 
szawa ausgeliehen wurden. $\mathrm{Zu}$ Vergleichszwecken wurde je eine Serie aus England (British Museum, Natural History) und aus Österreich (Zoologisches Museum in Wien) ausgeliehen. Man untersuchte ausserdem das aus den Karpaten stammende und sich in der Abteilung des Zoologischen Institutes der P. A. d. W. in Kraków befindende Materiaì.

Die aus der Kampinos-Heide stammenden Haselmäuse wurden wahrend der Durchsuchung von Vogelbrutkästen eingefangen. Fänge wurden im Juni, September u. Oktober durchgeführt.

Die lebendgefangenen Haselmäuse wurden nach Białowieża gebracht und dort gehalten.

Die Fangmethode für Schläfer in Vogelbrutkästen oder Fledermauskästen (S i d or o wi c z, 1958; 1959) scheint im Verhältnis zu dieser Säugergruppe die erfolgreichste zu sein. In der KampinosHeide, wo die Haselmaus eigentlich zahlreich auftritt (im Oktober 1958 waren $20 \%$ der Kästen durch sie belegt), konnte man trotz Anwendung verschiedener Fallen-Typen die Anwesenheit dieses Nagers nicht feststellen. Das ist eine nochmalige Bestätigung der bekannten Tatsache, dass man bei Benutzung nur einer einzigen Methode niemals Schlüsse über den quantitativen wie auch qualitativen Bestand der Säugerfauna eines bestimmten Gebietes ziehen darf.

Die Zeitspanne, in welcher wir Fänge durchführen, hat einen entschiedenen Einfluss auf die Anzahl und den Altersbestand der gefangenen Population. In dem in dieser Arbeit beschriebenen Material stellte ich das Alter grundsätzlich auf Grund des Abreibungsgrades der Backenzähne fest. In einigen Fällen kannte ich ihr genaues $\mathrm{Al}$ ter und zwar wenn ich sie von ihrer Geburt an hielt oder aus dem Nest nahm. Der Zustand der Vermehrungsorgane und KörperSchädel-Masse können ebenfalls gewisse Auskünfte über Alterbezeichnung liefern.

In dem aus dem Frühjahr aus dem Freiland stammenden Material besitzen wir unter den Überwinterlingen nur ausgewachsene, geschlechtsreife Individuen. Im Mai beginnen die ersten Jungtiere zu erscheinen, deren es allmählich immer mehr wird, so dass bereits ab August die Anzahl der Jungtiere und Alttiere fast dieselbe ist. Alle Jungtiere, welche im diesem Kalenderjahre geboren werden, sind noch nicht geschlechtsreif. Dieses sind nur Utberwinterlinge. Die beiden Altersgruppen - Jungtiere und Überwinterlinge 
unterscheiden sich unter einander sehr deutlich durch ihre Masse. Das gilt sowohl fur Körper- wie auch Schädelmasse. Dieser Zustand erhält sich bis zum Beginn des Winterschlafes der Haselmäuse.

Bei der Haselmaus-Haltung beobachtete ich, dass mit dem Beginn des Winterschlafes sich unter den "schlafenden" Exemplaren etwas wie eine Altersdurchgangsgruppe herausbildet, welche von der ,jungen" Gruppe stammt. Dieses sind junge Individuen, welche im Sommer oder Herbst geboren wurden und natürlich noch nicht geschlechtsreif sind, aber deren Schädelmasse sich von den Ausmassen der Individuen zu unterscheiden anfangen, welche im Herbst eingefangen wurden.

Es wirft sich hier die Frage auf, ob junge Haselmäuse während des Winterschlafes wachsen. Mein zu kleines Material erlaubt nicht, dieses Problem autorativ zu entscheiden. Zwei Tatsachen sind jedoch bekannt nämlich: a) - die im Herbst gefangenen Individuen teilen sich deutlich in zwei Gruppen, welche sich untereinander durch Schädelmasse und den Grad der Geschlechtsreife unterscheiden, b) - die Überwinterlinge haben ungefähr die gleichen Schädelmasse und sind alle geschlechtsreif.

Als einzig logische Erklärung dieser Erscheinung kann die Vermutung gelten, dass während des Winterschlafes oder gleich nach dem Erwachen eine Nivellierung der im Herbst bestehenden Unterschiede erfolgt, also dass die Jungtiere zu den Massen der erwachsenen Tiere anwachsen. Haselmäuse werden gleich nach dem Erwachen (oder noch am Ende des Winterschlafes) geschlechtsreif. Es ist möglich, dass diese beiden Prozesse der Reife und des Wachstums gleichzeitig erfolgen, aber das Erscheinen von „Durchgangsindividuen" dürfte vielmehr auf eine Verschiebung in der Zeit dieser beiden sich abwickelnden Prozesse hinweisen. Das Wachstum scheint jedoch der Geschlechtsreife voran zu gehen. Es scheint mir jedoch das ,,der Winteranwuchs" bei Tieren, welche in den Winterschlaf fallen, eine gewisse Gesetzmässigkeit hat; wenigstens bei denen, mit ähnlichen Massunterschieden in der Population im Herbst wie auch im Frühjahr, wie bei der Haselmaus und zwar bei Dryomys nitedula Pall., Sicista betulina Pall., Citellus suslica Gue ld.

Bei der Bearbeitung des aus der Kampinos-Heide stammenden Materiales, teilte ich es in drei Altersgruppen ein: I. Juvenes, II. 
Subadulti, III. Adulti. Uinter der Namenbezeichnung Subadulti verstehe ich junge überwinternde „Durchgangsindividuen”, Adulti bezeichnen Überwinterlinge und Juvenes diesjährige Jungtiere, vor dem Beginn des Winterschlafes.

Das ganze aus der Kampinos-Heide stammende Material wurde gewogen und gemessen. Die Schädel wurden trocken präpariert. Die Messungen wurden auf die allgemein übliche Weise durchgeführt, deren Methode unter anderen in der Arbeit von Wasilewski (1952) beschrieben worden ist. An dem Material aus Mokuszyn und den Museen London und Wien führte ich nur craniometrische Messungen durch.

\section{INDIVIDUELLE VERÄNDERLICHKEIT}

Bei der Beschreibung der Variabilität der Körpermasse stützte jch mich nur auf die Messungen der aus der Kampinos-Heide stammenden Haselmäuse. Das aus Mokuszyn stammende Material konnte ich leider nicht verwenden, da es anders vermessen worden war. Die Körpermasse in Einteilung auf drei Altersgruppen sind auf Tabelle 1 zusammengestellt. Die Altersunterschiede heben sich auf eine deutliche Weise hervor. Die Altersgruppe Subadulti nimmt eine deutliche Zwischenstellung ein. Dieses betrifft sowohl die Veränderlichkeitsamplituden wie auch die Mittelwerte. Die Körpermasse der Haselmäuse aus der Kampinos-Heide unterscheiden sich nicht von denen, welche von anderen Autoren für diese Art angegeben worden sind.

Tabelle 1.

Körperausmasse von $M$. avellanarius aus Dziekanów Leśny.

\begin{tabular}{|c|c|c|c|c|c|c|c|c|c|}
\hline \multirow[b]{2}{*}{ Altersklasse } & \multicolumn{3}{|c|}{ 1uv. } & \multicolumn{3}{|c|}{ subad. } & \multicolumn{3}{|c|}{ ad. } \\
\hline & min. & $\ddot{x}$ & $\max$. & min. & $\bar{x}$ & $\max$. & min. & $\bar{x}$ & $\max$. \\
\hline Kop?-Rump?länge & 57 & 65.0 & 74 & 65 & 68.6 & 74 & 69 & 75.1 & 80 \\
\hline Sohwanzlänge & 44 & 59.6 & 70 & 57 & 64.5 & 70 & 61 & 69.3 & 65 \\
\hline Hintexfussläng & 12 & 15.0 & 17 & 14 & 15.5 & 16.5 & 14 & 15.7 & 17 \\
\hline Ohrlänge & 9 & 10.0 & 11 & 9 & 10.8 & 12 & 11 & 11.4 & 12 \\
\hline $\mathrm{n}$ & \multicolumn{3}{|c|}{7} & \multicolumn{3}{|c|}{6} & \multicolumn{3}{|c|}{11} \\
\hline
\end{tabular}

Auf Tabelle 2 (craniometrische Messungen) ist das Material dargestellt, welches aus zwei Standorten stammt und zwar aus der Kampinos-Heide und den Rokitnosümpfen. Diese Standorte sind von einander zirka $350 \mathrm{~km}$. entfernt. Dieses Material in Einteilung 


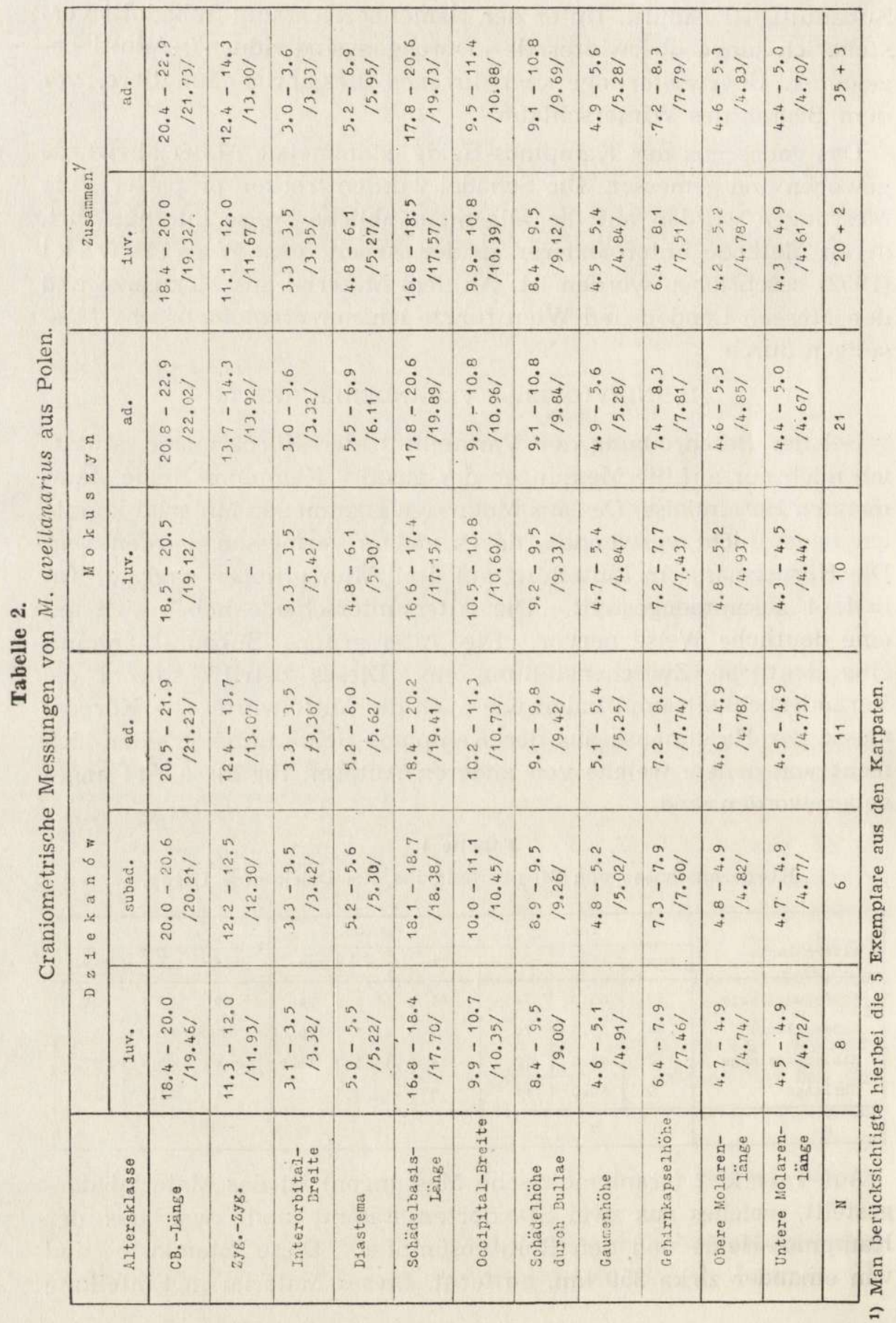


auf Altersgruppen (Klasse Subadulti nur für das Material aus der Kampinos-Heide) vergleichend, wollte ich feststellen, ob unter ihnen irgendwelche wesentliche Unterschiede bestehen, die auf geographische Variabilität deuten. In den letzten zwei Rubriken der Tabelle ist das ganze Material dargestellt, das noch mit Messungsergebnissen von 5 aus den Karpaten stammenden Haselmäusen ergänzt wurde. Aus dem Vergleich der craniometrischen Messungen der aus der Kampinos-Heide, Mokuszyn und den Karpaten stammenden Individuen sieht man keine wesentliche Unterschiede, obwohl die Entfernung dieser drei Standorte von einander sehr gross ist. Trotz verschiedener Herkunft macht dieses Material einen einheitlichen Eindruck. Dies betrifft sowohl den Bereich der individuellen Variabilität für jede Messung wie auch die Mittelwerte. Kleine Abweichungen in den Mittelwerten sind höchstwahrscheinlich durch die kleine Anzahl der Exemplare bedingt und nicht durch reelle Unterschiede. Die Veränderlichkeitsamplituden der einzeinen Messungen decken sich grundsätzlich. Wie es aus der Tabelle ersichtlich ist, deutet sich in der Klasse Subadulti ein Wachstum während des Winterschlafes an. Dies stimmt mit den oben beschriebenen Veränderungen in den Körpermassen der Haselmäuse überein.

Die Ergebnisse der Körper- $u$. Schädelmasse der Haselmäuse aus verschiedenen Landteilen weisen auf die Tatsache einer morphologischen Einheit des ganzen Materials hin. Angesichts dieser Angaben scheint es mir wahrscheinlich, dass die verglichenen Individuen trotzt der Herkunft von voneinander entfernten Standorten zu einer Unterart gehören.

Die untersuchten Haselmäuse haben eine charakteristische, einheitliche Färbung: Rücken und Schwanz gelbbraun - aschgrau, Bauch krem - gelb, auf der Kehle ein weisser Fleck, welcher bis zur Brust reicht und manchmal auf dem Bauche endet. Die Flanken und das Mäulchen sind etwas heller als der Rücken. Die individuelle Variabilität ist gross, und man kann in einer Serie einen graduellen Übergang von sehr hellen bis aschgrauen Individuen vorfinden. Diese Unterschiede verbleiben ebenfalls beim Winterkleid. Junge Individuen haben gewöhlich ein dunkleres Aussehen, mehr aschgrau,

Die Haare auf Rücken und Flanken sind in ihrem unteren Teile ciunkel-grau und im oberen ocker-gelb. 
Das Winterkleid ist viel dichter als das Sommerkleid, aber entgegen der Behauptung von Og nev (1947) unterscheiden sich die Winterhaare im Gegensatz zu den Sommerhaaren an Länge nicht. Es ist möglich, dass dies mit dem milderen Winter in Polen im Gegensatz zu dem aus der Gegend (Briansk, Moskva), woher O g n e v sein Material hernahm, in Verbindung stehen kann.

Über Termin und Verlauf des Haarwechsels bei der Haselmaus fehlt es völlig an Angaben in der Literatur. Im unseren Material befindet sich eine aufgezogene Serie an der man den Verlauf des winterlichen Haarwechsels überprüfen kann. Diese Erscheinung beginnt in den Monaten September u. Oktober. Gegen Ende des letztgenannten Monates sind schon fast alle Individuen im Winterkleid. Der Haarwechsel hat folgenden Verlauf: Er beginnt auf dem Rücken, greift auf die Flanken über, danach auf den Bauch und zuletzt auf das Mäulchen. Von den Käfig-Tieren stand noch ein Exemplar am 12 November im Haarwechsel; aber dies war schon seine letzte Phase, denn auf dem Rücken und den Flanken besass es schon sein Winterkleid. Da nun um diese Zeit herum Haselmäuse im Freiland, schon in den Winterschlaf gefallen sind, so sollte man vermuten, dass in Freilandbedingungen der Haarwechsel zu Anfang des Winterschlafes endet. Dies würde auf die Tatsache eines intensiven Verlaufes gwisser physiologischer Prozesse während des Winterschlafes hinweisen.

Weiterhin fehlt es an Angaben über den Frühjahrshaarwechsel. Die im Juni gefangenen Individuen sind schon im Sommerkleid, was darauf hinweist, dass der Frühlings-Haarwechsel gegen Ende des Winterschlafes oder gleich nach dem Erwachen verläuft. Angaben aus der Haltung können nicht massgehend sein, denn dort sind die Bedingungen künstlich und eine zu lange Zeitspanne ist schon seit dem Fang verflossen.

\section{BIOLOGIE DER HASELMAUS}

In unseren Bedingungen ist diese Art von Ende März - Anfang April bis zum September-Oktober aktiv. Die Zeit des Winterschlaf-Anfangs und des Erwachens im Frühjahr scheint mit den Witterungsverhältnissen verbunden zu sein, die in der gegebenen Periode herrschen. Ein Beispiel hierfür kann der warme Herbst 1958 sein, in dem man Haselmäuse in den Kästen (in ihnen überwintern sie nicht) bis zum 10. X. antraf. Genaue Angaben über mittel-russische Haselmäuse gibt Li c h a č e (1955) an, der ihre Sommer- 
aktivitätsperiode vom 14 . IV. bis 10 . X. bestimmt. Dieses deckt sich ganz mit unseren Beobachtungen aus der Kampinos-Heide.

Haselmäuse überwintern in unterirdischen Nestern ( $\mathrm{K}$ a $\mathrm{h} \mathrm{m}$ a n $\mathrm{n}$ u. Frisch, 1956; L i ch a č ev, 1955; W a chtend or f, 1951). Die Sterblichkeit soll während des Winterschlafes öfters recht hoch sein und nach I. i c h a č ev beträgt sie $80 \%$. Jungtiere vom Frühjahr sollen besser überwintern als diejenigen aus den Sommer- $u$. Herbstwürfen. Kahmann u. Frisch geben an, dass Haselmäuse oft einzein überwintern, ab und zu kann man jedoch eine viutter mit ihren Jungen finden. Meinen Beobachtungen nach findet man in der Regel im Spätherbst je einige Individuen in jedem Kasten. Meistenteils ist es ein Weibchen mit seinen Jungen, aber auch öfters einige Jungtiere ohne ein ausgewachsenes Exemplar. Als wir versuchten, sie in Gefangenschaft zu überwintern, indem wir jc zwei in einem Käfig hielten, starben doch recht viele. Als wir jedoch je 5 in einem Käfig untergebracht hatten, verringerte sich die Sterblichkeit wührend des Winterschlafes recht deutlich. Diese Angaben betreffen jedoch nur die Überwinterung in Käfigbedingungen, aber das kann auch auf eine Art von gruppenförmigem Einschlafen in einem Winternest hinweisen.

In der Kampinos-Heide beobachtete man Junge im Nest am frühesten am 31. V. Da nun die Schwangerschaft zirka 22-24 Tage dauert ( $\mathrm{Lich}$ a č e v, 1954), soll man annehmen, dass die Brunst in der ersten Maihälfte oder sogar Anfang April beginnt, folglich gleich nach dem Erwachen. Dies weist wiederum auf die Wahrscheinlichkeit unserer Beobachtungen betreffs der Zeit der Geschlechtsreifung hin, die sich gegen Ende des Winterschlafes oder gleich nach dem Erwachen vollzieht. Klimaverhältnisse üben einen grossen Einfluss auf die Vermehrung aus.

Es beginnen mit der Vermehrung Alttiere oder vorjährige Jungtiere aus dem Frühjahrswürfen.

Weibchen mit Jungen trifft man den ganzen Sommer lang bis zum Herbst hin an. (Ich sah ein Weibchen mit 5 blinden Jungen am 10 Oktober). Es wird angegeben, dass die Haselmaus während des Sommers zwei Würfe hat. Ich bin nicht in der Lage, mich über dies Thema auszusprechen, aber es scheint mir, dass diese Zahl zumindestens in einigen Jahren bedeutend höher sein kann. Es fällt jedoch keine Anzahlsteigerung von Weibchen mit Jungen während des Sommers auf, sodass wir in der Kampinos-Heide ein Bestehen von 
bestimmten Vermehrungsperioden nicht beobachten konnten. Die Vermehrung dauert den ganzen Sommer über. Lich a č e v gibt an, dass die Zeitspanne zwischen zwei Würfen 37-39 Tage beträgt und am kürzesten 28 Tage.

Die Wurfgrösse betrug in der Kampinos-Heide 1 bis 5 . Der Mittelwert von 33 beobachteten Würfen während zweier Jahre beträgt $3,55(3,43$ und 3,65$)$.

Frühere Würfe sind gewöhnlich grösser. Diese Tatsache ist interessant, denn es scheint, dass in der zweiten Sommerhälfte und im Herbst die Haselmaus bessere Nahrungsbedingungen hat. Dies ist mit den Angaben von $\mathrm{Lich}$ a če $\mathrm{v}$ übereinstimmend, welcher festgestellt hat, dass kleinere Würfe am Sommerende nicht voma Alter des Tieres sondern von der Jahreszeit abhängen. Nach der Meinung dieses Autors herrschen in den Würfen Männchen $(55,7 \%)$ vor. Der prozentsatzmässige Anteil der Jungen in der Population ist in den einzelnen Monaten verschieden. Die Ergebnisse der Kastenkontrolle aus der Kampinos-Heide aus zwei Jahren sind auf Tabelle 3 dargestellt. Dies sind nur Orientierungsangaben, denn das Alter wurde nur auf Grund der Grösse festgelegt und in gewissen Fällen konnten Individuen, die im Herbst für Erwachsene angeschen wurden, gut ausgewachsene Jungtiere aus den ersten Frühjahrwürfen gewesen sein. Jungtiere reifen im ersten Kalenderjahr ihres Lebens geschlechtlich nicht. Unsere Beobachtungen über die Populationsstruktur, die im Abschnitt Material und Methode besprochen worden sind, bestätigen ganz die Angaben von Lichač ev (1954).

Tabelle 3.

Zahlenmässige Zusammenstellung der Popuiation $M$. avellanarius aus der Kampinos Heide.

\begin{tabular}{|c|c|cccccccc|c|}
\hline \multicolumn{2}{|c|}{ Konat } & III & IV & V & VI & VII & VIII & IX & $X$ & Zusammen \\
\hline \multirow{2}{*}{1957} & 1uv. & - & - & 4 & - & 13 & 12 & 51 & - & 80 \\
\cline { 2 - 10 } & ad. & 7 & - & 10 & - & 8 & 14 & 41 & - & 80 \\
\hline \multirow{2}{*}{1958} & 1uv. & - & - & - & 15 & - & 13 & - & 19 & 51 \\
\cline { 2 - 10 } & ad. & - & 9 & - & 19 & - & 19 & - & 36 & 79 \\
\hline
\end{tabular}

Auf dem in der Kampinos-Heide beobachteten Geländestreifen, wo 300 Vogelkästen ausgehängt waren, traf man auf fast keine Haselmausnester ausserhalb derjenigen, welche in den Vogelkästen vorhanden waren. Verhältnismässig oft beobachtete man, dass Ha- 
selmäuse Vogelkästen bezogen in denen sich schon Vogelnester befanden (hauptsächlich Parus sp. und Muscicapa sp.). Diese Nester wurden zerstört, wobei Eier oder Vogelbrut des öfteren herausgeworfen wurden. Die Haselmaus baute ihr Nest unter dem schon vorhandenen Vogelnest, das sie auf ihrem Neste immer höher hob, aber mit dem Erreichen des Fluglochniveau's durch das Vogelnest wurde es herausgeworfen.

Es scheint, dass die Haselmäuse keine beträchtlichen Vogelfeinde haben. In dem Gewolle von Raubvögein fand man keine Reste von ihnen. (Man untersuchte ungefähr 50.000 Stück Eulengewölle).

Die Haselmaus ist von der Abend- bis zur Morgendämmerung aktiv (Ostermann, 1956). Während des Tages verbirgt sie sich im Kasten oder im Nest. Aus dem Kasten vertrieben, flüchtet sie in die Baumkione oder auf die Erde, wo sie versucht, sich im Fallaub zwischen den Baumwurzeln zu verstecken. Sie lässt sich jedoch leicht fangen. Als Nahrung dient ihr grundsätzlich die Pflanzenkost (W a chtendorf, 1951). In der Gefangenschaft fressen sie Obst, Samen, Haselnüsse (nur geöffnete). Sie sind sehr leicht zu halten.

Die Haselmaus ist ein Waldtier, was alle Autoren übereinstimmend feststellen, indem sie ihr Auftreten mit gewissen Waldtypen verbinden. Ognev (1947) gibt an, dass man sie in Laubwäldern mit Beimischung von Fichen, Linden und sogar Erlen u. Tannen vorfinden kann. In solchen Wäldern wachsen Haselnussträucher. V i$n \circ \mathrm{gradov} u$. Grom ov (1958) unterstreichen, dass sie speziell Eichenwälder bevorzugt, da im Unterholz ausser den Haselnusssträuchern noch Ahlkirschbäume und Schneeballsträucher vorkommen. Ge ptner (1950) stellt fest, dass die Haselmaus seltener in Mischwäldern mit Beimischung von Kiefern u. Tannen vorkommt. Lichačev $(1954,1955)$ fing in den Gegenden von Moskau Haselmäuse in Eichen-Lindenwäldern und Espen-Birkenwäldern. Er unterstrich, dass die dort hausende Art den Jung $u$. Mittelwald bevorzugt und sehr selten im alten Hochwald vorkommt. In Misch- $u$. Nadelwäldern tritt sie fast garnicht auf.

Pop ov (1955) gibt an, dass die Haselmaus in Bulgarien in Laubu. Mischwäldern mit Beimischung von Haselnussträuchern, Ahorn, Esche und Eiche $u$. Linde vorkommt.

Für Frankreich notiert sie D uchert (1950) in der Haute Savoie (Grenzland mit der Schweiz) in Tannenwäldern. W a c h te nd or f (1951) fing Haselmäuse auf Holzschlägen, auf denen Erdbeeren, 
Himbeeren u. Brombeeren wuchsen, in jungen Laubmischwäldern mit Eichen u. Haselnussträuchern und in Tannenwäldern. Die Beobachtungen führte dieser Autor in den Alpen durch. Weiterhin stellen Kahmann u. Frisch (1950) fest, dass diese Art zumindestens in den Alpen das Auftreten von Haselnussträuchern in den Regionen, wo sie vorkommt, nicht benötigt. In grösserer Anzahl haust sie in jungen Buchen- $u$. Tannenwäldern, und gerade diese Bäume bildenden für sie charakteristischen Biotop. In Hochwäldern kommt sie eigentlich nicht vor. Diese Ansicht bestätigt G r o d z i nsk i (1958), der Haselmäuse nur in jungen Laubwäldern antraf, die in den Karpaten auf einem vernichteten Wald vom Typus Fagetum carpathicum wuchsen. In der Tatra tritt sie an Waldbächen auf mit Ausnahme der alpinen Region (Rosicky u. Kratochvil, 1955).

In der Kampinos-Heide, wo ich Haselmäuse fing, kommt sie in folgenden Biotopen vor: Circeo-Alnetum, Querceto-Carpinetum, Pinetum typicum und Pineto-vaccinietum uliginosi. Das Durchschnittsalter des Baumbestandes betrug 30-40 Jahre. Im Unterholz wachsen Haselnussträucher, Frangula, Erlen, Eichen und Kie$f \in r n$. All das erwähnte Gelände ist ziemlich feucht. Die Haselmäuse wohnten in Vogelkästen, die in der Nähe von Stegen, Pfaden und lichtungen ausgehängt worden waren. Ich fand dagegen keine Haselmäuse in dem auf demselben Gelände auftretenden trockenen Kiefernhochwald, der als Unterholz Eiche u. Haselnussträucher besass. Ein Haselmausnest wurde sogar auf einem Wacholderstrauch angetroffen.

Wie es sich aus dem Schrifttum und den Beobachtungen des Autors ergibt, ist das Auftreten der Haselmaus mit dem Laubwald oder Mischwald eng verbunden. Am typischsten für sie ist der Jungwald oder der im mittleren Alter stehende Wald aber mit dichtem Unterholz. In solchem Walde findet sie optimale Existenzbedingungen. In Wäldern mit altern Baumbestand oder Hochwäldern kommt sie fast garnicht vor. Sie kann in sehr feuchten Wäldern sogar sumpfigen wie auch in Gebirgswäldern auftreten. Sehr gern bemächtigt sie sich der Vogelkästen. Angesichts der erwähnten Tatsachen ist es verständlich, warum die Haselmaus in dem Białowieżaer Nationalpark nicht auftritt; sie findet einfach in den Altbaumkomplexen keine entsprechenden Bedingungen.

Was das vertikale Siedlungsgebiet dieser Art betrifft, so findet 


\begin{tabular}{|c|c|c|c|c|c|c|c|c|c|c|c|c|}
\hline $\begin{array}{l}\dot{\alpha} \\
\vdots \\
\dot{\sigma} \\
\dot{j} \\
\dot{j} \\
\dot{b}\end{array}$ & 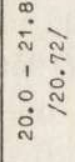 & 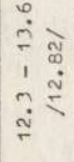 & $\begin{array}{l}9 \\
\dot{p} \\
1 \\
\dot{m} \\
\dot{m}\end{array}$ & 11 & 1 & 1 & 1 & 1 & ' & 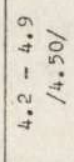 & I & $\Rightarrow$ \\
\hline 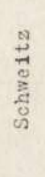 & 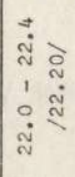 & 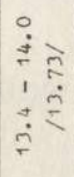 & 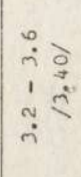 & 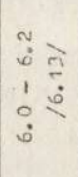 & ' & 1 & I & ' & 1 & 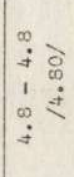 & 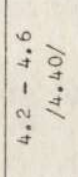 & m \\
\hline 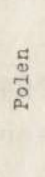 & 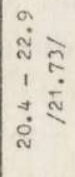 & 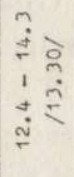 & 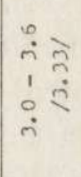 & 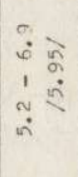 & 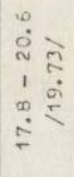 & 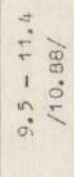 & 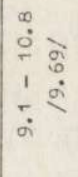 & 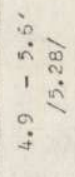 & 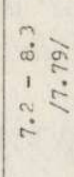 & 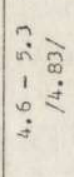 & 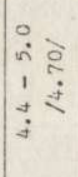 & $\approx$ \\
\hline 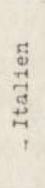 & 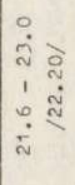 & $\begin{array}{l}\infty \\
\dot{0} \\
\dot{0} \\
1 \\
\dot{j} \\
\dot{f} \\
\dot{f}\end{array}$ & 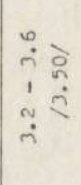 & 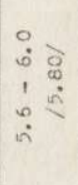 & 1 & 1 & ' & ' & , & $\begin{array}{l}0 \\
\dot{0} \\
1 \\
0 \\
\infty \\
\dot{0} \\
\dot{i}\end{array}$ & $\stackrel{\vdots}{i}$ & + \\
\hline 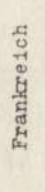 & $\begin{array}{l}\infty \\
\stackrel{n}{n} \\
\dot{i} \\
1 \\
0 \\
\dot{i} \\
\dot{N}\end{array}$ & 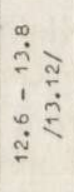 & 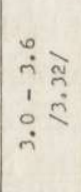 & 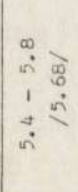 & 1 & I & 1 & 1 & & 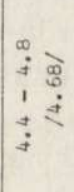 & 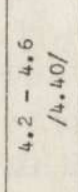 & n \\
\hline 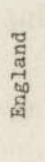 & 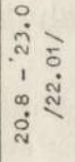 & 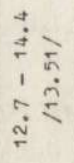 & 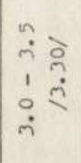 & 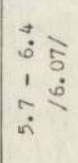 & 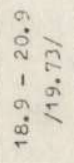 & 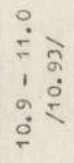 & $\begin{array}{l}0 \\
\dot{0} \\
i \\
1 \\
0 \\
\vdots \\
0 \\
0\end{array}$ & 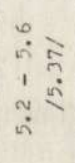 & 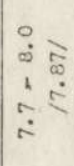 & 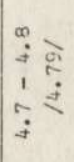 & 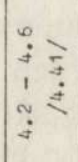 & $\infty$ \\
\hline 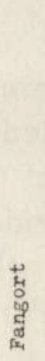 & 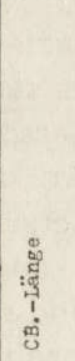 & 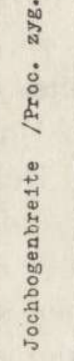 & 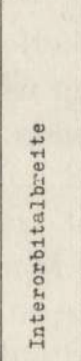 & 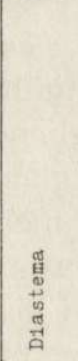 & 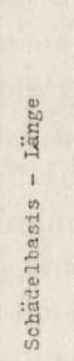 & 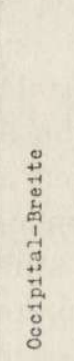 & 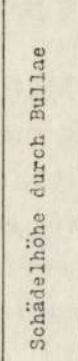 & 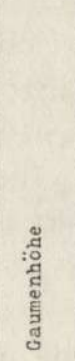 & 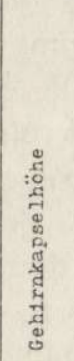 & 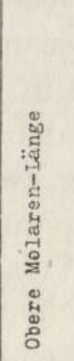 & 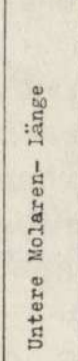 & $=$ \\
\hline
\end{tabular}


man sie bis zur Waldgrenze. Nach Rosicky u. Kratochvil (1955), trifft man Haselmäuse bis zur Höhe von $1700 \mathrm{~m}$. G e r b e r (1952) gibt die Höhe von $900 \mathrm{~m}$. an und $\mathrm{Mohr}$ (1950) sogar bis $2000 \mathrm{~m}$. über dem Meeresspiegel. Diese Angaben dienen nur zur Orientierung und hängen von der Höhe ab, auf der die obere Waldgrenze in den gegebenen Gebirge vorkommt.

\section{SYSTEMATISCHE STELLUNG DER EINHEIMISCHEN VERTRETER DER GATTUNG MUSCARDINUS K A UP.}

Die Haselmaus tritt auf einem sehr grossen Gebiet auf und zwar von England und Centralschweden bis zu den Pyrenäen, von Sizilien bis Kasań (U. d. SSR) und Klein-Asien (O gn e v, 1947; Van den Brink, 1956; Dar ling to n, 1957). Fossile Schläfer sind in Europa eine gewöhnliche Erscheinung (S i m p s o n, 1945). Auf dem ganzen Gebiet ihrer Verbreitung hat diese Art 5 geographische Formen ausgebildet. Zwei von diesen Formen - M. avellanarius niveus Altobello und $M$. pulcher Barrett - Hamilton treten nur in Italien auf (zusammen mit $M$. avellanarius avellanarius L.). M. avellanarius zeus $\mathrm{Ch}$ a worth $-\mathrm{Muster}$ tritt in Griechenland und M. trapezius Mille r in Klein-Asien auf. Nach E 11 e r m a n n (1940) kommt auf dem übrigen Gebiet $M$. avellanarius avellanarius $\mathrm{L}$. vor, der aus Mittel-Schweden beschriehen worden ist. Elle rmann hält die unter dem Namen von $M$. avellanarius anglicus $\mathrm{B}$ a r ret $\mathrm{t}-\mathrm{H}$ a milt on beschriehene Form für Synonym von $M$. avellanarius avellanarius. Eine ähnliche Ansicht sprach Milier (1912) aus:

...,,Skins from different parts of the range of Muscardinus avellanarius show practically no individual variation. Adults are darker and more richly coloured that the joung, thought difference is slight. British specimens are not distinguishable from these taken in Switzerland and Italy".

Die craniometrischen Messungen (Tabelle 4) weisen ebenfalls darauf hin. Das ganze Material, gleichgültig woher es stammt, macht den Eindruck eines vielmehr Ausgeglichenen. Unterschiede in der Färbung zwischen Individuen aus England und Polen sind nicht ersichtlig und praktisch genommen nicht zu unterscheiden, was schliesslich damit übereinstimmt, was Mille r angegeben hat. Unterschiede in den craniometrischen Messungen sind so unwesentlich, dass man sie mit der kleinen Individuenanzahl begründen kann. Wenn man sich auf das Fehlen von Unterschieden zwischen 
Individuen aus verschiedenem Gelände stützt, so scheint es mir, dass man annehmen darf, dass alle zu einer Unterart gehören.

Angesichts der erwähnten Tatsachen und unserer bisherigen Kenntnis von dieser Art, bin ich der Meinung, dass in Polen Muscardinus avellanarius avellanarius $\mathrm{L}$. auftritt.

\section{ZUSAMIMENFASSUNG}

Im Ergebnis der morphologischen Analyse des aus der KampinosHeide und den Rokitnosümpfen stammenden Materiales und des Vergleichmateriales aus England u. Österreich, sowie der Angaben aus dem Schrifttum stellte man die gänzliche Einheitlichkeit des verglichenen Materiales fest. Alle verglichenen Individuen gehören zur Unterart Muscardinus avellanarius avellanarius L.

Bei der Haltung junger Haselmäuse während des Winters vermutet der Autor die Erscheinung des Wachsens junger Individuen während des Winterschlafes beobachtet $\mathrm{zu}$ haben, die in dieser Zeit die Masse von erwachsenen Stücken erreichen. Dieses betrifft sowohl Körper- wie auch Schädelmasse.

Es wurde der Verlauf des herbstlichen Haarwechsels beschrieben, der im September u. Oktober stattfindet, aber schon während des Winterschlafes endet. Auf Grund von Freilandbeobachtungen und desgleichen in der Haltung wurde eine Reihe von Tatsachen beschrieben, die mit Winterschlaf, Vermehrung und Biotopcharakteristik der Haselmaus in Verbindung stehen.

Institut für Säugetierforschung in Białowieża,

Polnische Akademie der Wissenschaften.

\section{SCHRIFTTUM}

1. Belke, G. - Mastodologia czyli historya naturalna zwierząt ssących. Wilno, 1848.

2. Brin k, van den, F. H. - Die Säugetiere Europas. Paul Parey. Hamburg - Berlin, 1956.

3. Darlingt on, J. Jr. - Zoogeography: the Geographical Distribution of Animals. J. Wiley: $675+$ XIV. London, 1957

4. Duchert, D. - Observations de muscardins en France. Mammalia, 14:183. Paris, 1950.

5. Eisentraut, M. - Beobachtungen über den Winterschlaf der Haselmaus. Ztschr. Säugetierk., Vol. 4:213--239. Berlin, 1929. 
6. Elle rman, J. R. .- The Families and Genera of Living Rodents, Vol. 1. Brit. Mus.: $612-626$. London, 1940.

7. Frisch, O. -- Fluchtverhalten der Haselmaus (M. avellanarius L.). Ztschr. Tierpsychol., Vol. 8:368-369. Berlin, 1951.

8. Gerber, R. - Nagetiere Deutschlands. Die Neue Brehm-Bücherei, No. 27. Geest \& Portig. Leipzig, 1952.

9. Grodzinski, W. - The Succesions of Small Mammal Communities on an Overgrown Clearing and Landslip in the Western Carpathians. Bull. Acad. Polon. Sci. II, Vol. 6: 431-439. Warszawa, 1958.

10. Geptner, W. G. et all. - Vrednye i poleznye zvieri polezaščitnych nasaždenij. 1950.

11. K a h mann, H. \& v. Fris c h, O. - Zur Ökologie der Haselmaus (M. avellanarius L.) in den Alpen. Zool. Jb. (Syst.), Vol. 78:531-546. Jena, 1950.

12. Ka rpiński, J. J. - Materiały do znajomości ssaków Puszczy Białowiejskiej. Rocz. Nauk. Leśnych, Vol. 14, 151:125-162. Warszawa, 1954.

13. Lichačev, G. N. - Razmnoženije i čislennost orešnikovoj soni. Zool. Zhurn., Vol. 33, 5, Moskva, 1954.

14. Lichačev, G. N. - Vremja probuždenija i charakter kolebok orešnikovoj soni. Bull. Mosk. Obšč. Ispyt. Prir., Vol. 60, 4:123-124. Moskva, 1955.

15. Miller, G. S. - Catalogue of the Mammals of Western Europe.... Brit. Mus. London, 1922.

16. Mohr, E. Die freilebenden Nagetiere Deutschlands. G. Fischer: 152+ VIII. Jena, 1950

17. Ognev, S. I. - Zveri SSSR i priležaščich stran. AN SSSR. Vol. 5: 532-545. Moskva-Leningrad, 1948.

18. Ostermann, K. - Zur Aktivität heimischer Muriden und Gliriden. Zool. Jb. (Syst.), Vol. 66:335-389. Jena, 1956.

19. Pietruski,, S. - Historya naturalna zwierząt ssących dzikich galicyjskich. Lwów, 1853.

20. Pietruski, S. - Dwa odczyty o niektorych rzadszych krajowych zwierzętach ssących. Lwów, 1864.

21. Popov, W. - Vrednite grizači i borbata s tjach. Zemizdat. Sofia, 1955.

22. Rosicky, B. \& Kratochvil J. - Drobni ssavei Tatranskoho narodniho parku. Ochrana Prirody, Vol. 10, 2:3-16. Praha, 1955.

23. Sidorowicz, J. - Some notes on the Edible Dormouse (Glis glis L.) in Poland. Acta Theriol. Vol. 2, 14:292-295. Białowieża, 1958.

24. Sidorowicz, T. - The Forest Dormouse (Dryomys niteduia Pa11.) in Białowieża, 1959. Acta Theriol. Vol. III: 17-26. Białowieża, 1959.

25. Simpson, G. G. - The Principles of Classification and a Classification of Mammals. Bull. Amer. Mus. Nat. Hist. Vol. 85:1-350. New York, 1945.

26. Skuratowicz, W. - Klucz do oznaczania krajowych zwierząt ssących. Księgarnia Akademicka. Poznañ, 1947.

27. Skura towicz, W. - Badania nad fauną ssaków Zamojszczyzny. Fragm. Faun. Mus. Zool. Polonici, Vol. 5, 15:233-292. Warszawa, 1948. 
28. Stronczyriski, K. - Spis zwierząt ssących Kraju Polskiego i pogranicznych. Warszawa, 1839.

29. Tomilin, A. G. - Nekotoryje dannyje o termoregulacji u orešnikovoj soni (Muscardinus avelianarius L.). Zool. Zhurn. Vol. 37, 1: Moskva, 1958..

30. Turov, S. S. - Predvaritelnyje zamečanija o faunie mlekopitajuščcich Belovežskoj Pušči. Uč. Zap. Mosk. Gorod. Pied. Inst., Vol. 38: 5--12. Mo. skva, 1955.

31 T yzenhauz, K. - Catalogus Avium et Mammalium... (w oprac. J. Domaniewskiego). Fragm. Faun. Mus. Zool. Polonici, Vol. 1, 10: 209-288. Warszawa, 1931.

32. Wa chtendorf, W. - Beiträge zur Ökologie und Biologie der Haselmaus (M. avellanarius) in Alpenvorland. Zool. Jb. (Syst.), Vol. 80: 189c04, Jena, 1951.

33. Wasilcwski, W. - Badania nad morfologią Clethrionomys glareoius glareolus Schreb. Ann. Univ. M. Curie-Sklodowska, Sect. C, Vol. 7, 3: 119-211. Lublin, 1952.

34. Vinogradov, B. S. \& Gromov, I. M. - Gryzuny fauny SSSR. AN SSSR. Moskva, 1952.

35. Zimmermann, K. - Exkursions Fauna von Deutschland; Wirbeltiere, Manmalia. Volk u. Wissen: 300. Berlin, 1955.

\section{STRESZCZENIE}

W wyniku analizy morfologicznej materiału pochodzącego z Puszczy Kampinowskiej i Polesia, oraz materialów porównawczych z Anglii, Austrii i danych z literatury, stwierdzono całkowitą jednorodność porównywanego materiału. Wszystkie porównywane osobniki należą do podgatunku Muscardinus avellanarius avellanarius $\mathrm{L}$.

Hodując przez zimę młode orzesznice, autor zaobserwowal zjawisko wzrostu młodych okazów w czasie snu zimowego, które w tym czasie osiągają wymiary sztuk dorosiych. Dotyczy to zarówno wymiarów ciała jak i czaszki.

Zostal opisany przobieg linki jesiennej, która ma miejsce we wrześniu i październiku a kończy się już w czasie snu zimowego. Na pođstawie obserwacji terenowych i z hodowli zostało opisane szereg faktów związanych ze snein zimowym i charakterystyką biotopu orzesznicy.

Państwowe Wydawnictwa Naukowe \& Warszawa $1959 \mathrm{r}$. Naklad 1505 egz. Ark. wyd. 1.232. Maszynopis otrzymano 15.VI.1959 r. Podpisano do druku 23.X.1959 r. Druk ukończono 15.XI.1959 r. Papier druk sab. k1. III, 80 gr. Format B-5

Bialostockle Zaklady Graficzne. Zam. 1838, Cena 6 zl. 


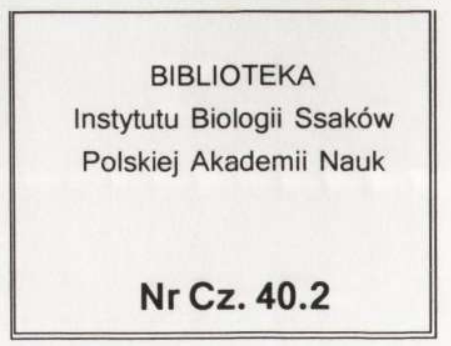

